\title{
Developing an Ontology for Documenting Adverse Events While Avoiding Pitfalls
}

\author{
Stefanie NEUENSCHWANDER ${ }^{\text {a }}$, Patricia ROMAO ${ }^{\text {a }}$, Jürgen HOLM ${ }^{\text {a }}$ \\ and Murat SARIYAR ${ }^{\mathrm{a}, 1}$ \\ ${ }^{a}$ Bern University of Appl. Sciences, Department of Medical Informatics, Switzerland
}

\begin{abstract}
Ontologies promise more benefits than terminologies in terms of data annotation and computer-assisted reasoning, by defining a hierarchy of terms and their relations within a domain. Here, we present central insights related to the development of an ontology for documenting events during interoperative neuromonitoring (IOM), for which we used the Basic Formal Ontology (BFO) as an upper-level ontology. This work has the following two goals: to describe the development of the IOM ontology and to guide the practice with respect to documenting of biomedical events, as available ontologies pose difficulties on certain issues. We address the following issues: (i) differentiate between the sets \{documentation, identification, continuant\} and \{explanation, understanding, occurrent $\}$ as we had problems in applying the available ontology of adverse events, (ii) covering diseases and injuries in a consistent way, and (iii) deciding on which level to define relations.
\end{abstract}

Keywords. Adverse events, BFO, clinical documentation, intraoperative neuromonitoring, phenomenology

\section{Introduction}

Medical interventions related to the use of certain medical devices and products might cause unintended adverse events (AEs) [1]. Frequently, drug-related AEs are addressed in the literature [2], but AEs caused by surgery processes also generate significant burdens in terms of mortality and associated healthcare costs [3]. In order to capture the type of AEs, it is instrumental to document the results of associated health interventions in an unequivocal and standardized manner. For this purpose, standard terminologies [4] and ontologies [5] have been developed.

The main terms used in ontological hierarchies are universals, i.e., they represent what certain entities have in common [6]. It is frequently difficult to capture what such common characteristics are. For example, in the ontology of adverse events (OAE [1]), an $\mathrm{AE}$ is defined as "a pathological bodily process that occurs after a medical intervention", which also applies to processes after treatment of a chronic disease. Main motivation for this work is related to such ambiguous definitions and additional problems in the OAE, which we have encountered during the development of our ontology.

Our use case is the documentation of adverse events during interoperative neuromonitoring (IOM), for which we used the Basic Formal Ontology (BFO) as an

${ }^{1}$ Corresponding Author, Murat Sariyar, Bern University of Applied Sciences, Quellgasse 21, CH2502 Biel/Bienne, Switzerland; E-mail: murat.sariyar@bfh.ch. 
upper-level ontology [7, 8]. IOM is the continuous measurements of neural electrical activity during neurosurgery to prevent postoperative deficits. BFO is a small upper-level ontology used by over 250 ontology-driven projects worldwide, especially for the biomedical domain. The fundamental distinction of BFO is between continuants, i.e., those entities that persist through time and are fully present at any point in time at which they exist at all (e.g., material entities such as syringes), and occurrents, i.e., those entities that unfold themselves in time and are present at any time only through their parts, e.g., my whole life with my childhood as one part at a certain time span.

Barry Smith has contributed central methods and notions to the scientific field of ontology. By consolidating his philosophical insights into the work on the BFO, he allowed many researchers to use sound concepts without having to understand the philosophies behind them. However, our work shows that it is sometimes crucial to understand the philosophical theories to make and justify certain decisions in practice. Without reference to philosophical works, it would have been difficult to detect the essential reason why the ontology of adverse events was not appropriate for us.

This work has the following two goals: to describe the development of the IOM ontology and to guide the practice with respect to documenting of biomedical events, as available ontologies pose difficulties on certain issues. We address the following issues: (i) differentiate between the sets \{documentation, identification, continuant\} and \{explanation, understanding, occurrent\}, (ii) covering diseases and injuries in a consistent way, and (iii) deciding on which level to define relations.

\section{Methods}

Guiding the practice required to adequately justify certain decisions during ontology development. For this purpose, the philosophical backbone of BFO were briefly analyzed and central implication for the practice was deduced from it.

To develop the IOM ontology, we first collected a list of relevant terms. In the second step, we defined a hierarchy between these terms through is_a (taxonomy) and part_of relations (mereology). In this step, the construction of the taxonomy and the reuse of existing ontologies was facilitated by referring to an upper-level ontology. Finally, further relations between two terms were defined (i.e., they are connected through a predicate), e.g., bearer_of (dependence) or is_connected_to (topology), and the terms themselves received further clarification by providing data properties, e.g., has_date or has_color. We used Webprotégé as development environment, Ontofox for reusing available ontologies, JavaFX for the frontend, and Apache Jena for the backend.

\section{Results}

\subsection{Philosophical orientation in the IOM ontology}

BFO has a philosophical orientation like other upper-level ontologies such as DOLCE and SUMO. It is strongly inspired by the phenomenologist Roman Ingarden, who advocated an immanent-realist view: there are mind-independent things in the spatiotemporal world and universals (kinds, properties, and relations), which capture them [9]. To put it in another way, the things are not just what we can sensually perceive, but also composed of categories, e.g., being a continuant. However, there is no direct 
experience of things in terms of sensual and categorical properties. Experience is always intentionally directed towards things, through thoughts, images, desires, etc. A consequence is that we are always dealing with objects-as-intended (noematic structure), i.e., our intentional structure should be accounted for, when describing things.

During our ontology development it became crucial to consider the intentional structure for inferring following insight: continuants are associated with the intention to identify things, while occurrents are related to the intention to retrace something. Hence, if we want to identify a disease, we handle it like a continuant, if we want to retrace it (i.e., understand the course of the disease and its events), it functions as an occurrent. In other words, it is not useful to subsume events to the occurrence class if the goal of the final ontology is to facilitate documentation. These leads to the differentiation between the sets \{documentation, identification, continuant\} and \{explanation, understanding, occurrent\}.

\subsection{Results and decisions in the IOM ontology}

The IOM ontology is available via a user-friendly JavaFX frontend (access to the code at https://gitlab.ti.bfh.ch/neues4/IOMDO is granted upon request). It is not used for realworld documentation yet, even though some preliminary user tests were promising. Most of the terms in the IOM ontology were collected in a previous work, which lacked a taxonomy and relations [10]. For documenting the possible events during IOM with the help of an ontology, we relate those events to measurement data of evoked potentials and to induced postoperative deficits. With respect to the handling of events in our ontology, we initially relied on the OAE. However, in [1], it is stated that the ontology "allows the development and application of new analysis methods to better understanding the mechanisms of adverse events associated with or induced by different medical interventions", which means that the primary intention is to understand or retrace the processes that lead to adverse events [11].

What was the problem for the IOM ontology? In the adapted process-oriented view of the OAE, instances were of the form "Process P02 taken place on Sunday 10:00 pm leading to bleeding". Without further characterization of the processes in terms of dynamics and concrete components, such instances were useless for documenting events and associated data. In the gene ontology, for example, the process "hexose biosynthetic process" is integrated into a hierarchy of processes in order to understand the components of the metabolic processes. The aim is to retrace the sub-processes of the metabolic processes in general, not to identify concrete processes within an individual, which would be difficult anyway. In our initial approach, the difference of our perspective and that of OAE was overlooked. Even if one can combine both perspectives, the complexity of the resulting ontology would be very high. For documenting events, we finally used the generally dependent continuant term of BFO, as it has been used by the Ontology for General Medical Science (OGMS) for diagnoses [12]. Events are subsumed under the class 'information content', as we want to identify them, and for this goal, there is no benefit in referring to them additionally or alternatively as processes.

Regarding the classification of injuries, we relied again on the OGMS diagnosis and on the disposition concept for diseases. In OGMS, a disease "is a disposition to undergo pathological processes that exists in an organism because of one or more disorders in that organism". First, we decided that injuries belong to the category of disease, even though they are not dispositions per se. There are two reasons for this decision: (a) ICD10 has injuries as part of its categories ( $\mathrm{S} 00-\mathrm{T} 88)$, (b) the effects of an injury depend on 
the disposition of an individual. Second, we used injury diagnoses as entities with content, for the same reasons as for the classification of events in our ontology.

Considering relations, we could define all relations at the class level of our ontology or rely on other ontologies. The second approach means that we have to import the corresponding ontologies if we want to enable inferences based on reasoners. We decided to import the relation ontology because it provides a BFO-based collection of relations intended for standardization across different biomedical ontologies, e.g., we could use the "before", "has_phenotype", "has_participant" and other relations out-of-the-box [13].

\section{Conclusion}

Developing and understanding the distinction between different intentional structures (identification versus explanation) helped us to make informed decisions with respect to the classification of the terms "events" and "injuries" in an ontology destined to facilitate documentation. In contrast to the OAE, our ontology can be used off-the-shelf for documentation. Our insights have implications for several discussion related to the use of BFO. For example, uncertainties regarding the increase of complexity, when SNOMED CT is based on BFO can be reduced by referring to the distinction mentioned in the previous paragraph. For example: When diagnosing a patient with asthma in a healthcare setting, it is always an act of identifying the disease, not to retrace it. The related asthma attacks contribute to the characterization of asthma by being referred to them as 'information contents' rather than processes. The processes are relevant to the biomedical researcher who wants to better understand the disease by investigating certain dynamic patterns and who is, in most cases, not interested in individual cases.

\section{References}

[1] He Y, Sarntivijai S, Lin Y, et al. OAE: The Ontology of Adverse Events. J Biomed Semant 5 (2014), 29.

[2] Huang H-C, Wang C-H, Chen P-C, et al. Bibliometric Analysis of Medication Errors and Adverse Drug Events Studies. J Patient Saf 15 (2019), 128-134.

[3] Classen DC, Pestotnik SL, Evans RS, et al. Adverse Drug Events in Hospitalized Patients: Excess Length of Stay, Extra Costs, and Attributable Mortality. JAMA 277 (1997), 301-306.

[4] Breder CD. A Method for Retrieval of Adverse Event Terms in Clinical Trial Databases Using Standardised MedDRA Queries. Pharm Med 30 (2016), 103-108.

[5] Guo A, Racz R, Hur J, et al. Ontology-based collection, representation and analysis of drug-associated neuropathy adverse events. J Biomed Semant 7 (2016), 29.

[6] Armstrong DM. A Theory of Universals: Volume 2: Universals and Scientific Realism. CUP Archive, 1980 .

[7] Arp R, Smith B, Spear AD. Building Ontologies with Basic Formal Ontology. MIT Press, 2015.

[8] Smith B. Ontology. In: Floridi L (ed) The Blackwell Guide to the Philosophy of Computing and Information. Blackwell Publishing Ltd, pp. 153-166.

[9] Johansson I. Proof of the Existence of Universals - and Roman Ingarden's Ontology. Metaphysica 10 (2009), 65-87.

[10] Zbinden C, Strickler M, Sariyar M, et al. A Protocol Entry Catalog for Intraoperative Neuromonitoring Steps Towards an Ontology. Stud Health Technol Inform 272 (2020), 318-321.

[11] Yu H, Nysak S, Garg N, et al. ODAE: Ontology-based systematic representation and analysis of drug adverse events and its usage in study of adverse events given different patient age and disease conditions. BMC Bioinformatics 20 (2019), 199.

[12] El-Sappagh S, Franda F, Ali F, et al. SNOMED CT standard ontology based on the ontology for general medical science. BMC Med Inform Decis Mak 18 (2018), 76.

[13] Guardia GD, Vêncio RZ, de Farias CR. A UML profile for the OBO relation ontology. BMC Genomics 13 (2012), S3. 